\title{
SYSTEM SIMULATION FOR HYDRAULIC EXCAVATOR
}

\author{
Kazuhide MAEHATA \\ Development Sect., New Products Development Dept., Hydraulic Components Operations \\ KYB Corporation \\ 1-12-1 Asamizodai, Sagamihara-shi, Kanagawa-ken, 228-0828 Japan
}

\begin{abstract}
The various hydraulic components for excavator have been developed in KYB Corporation. In early stage of the product development, we usually use calculation of static characteristics, but we do not use calculation of dynamic characteristics for the system. The dynamic characteristics of the system are tuned using actual system in middle stage of the product development. Therefore it is often necessary to extend the product development time. In order to decrease the development time, we developed a simulation model of excavator system for dynamic characteristics calculation. This paper shows case studies of the system simulation for hydraulic excavator applied to the digging-turning-loading cycle analysis.
\end{abstract}

\section{KEY WORDS}

Simulation, Excavator, SIMULINK, ADAMS

\section{SIMULATION MODEL}

Figure 1 shows the structure of the simulation model. The simulation model consists of body model for calculating motion of excavator, and hydraulic circuit model for calculating behaviors of hydraulic circuit.

The actuator force is transmitted from the hydraulic circuit to the body.

The actuator speed and length of the body are fed back to the hydraulic circuit.

In this case, the hydraulic circuit model is modeled on SIMULINK, a control system analysis software, and the body model is represented on ADAMS, a mechanical system analysis software.

Figure 2 shows the body model.
The body model on ADAMS consists of moving parts and constraints (joints). Each moving part has polygon-based geometry to allow easier and quicker modeling. All parameters, such as geometry dimensions and mass properties, can be obtained via actual measurements, relevant technical papers and $3 \mathrm{D}$ CAD data.

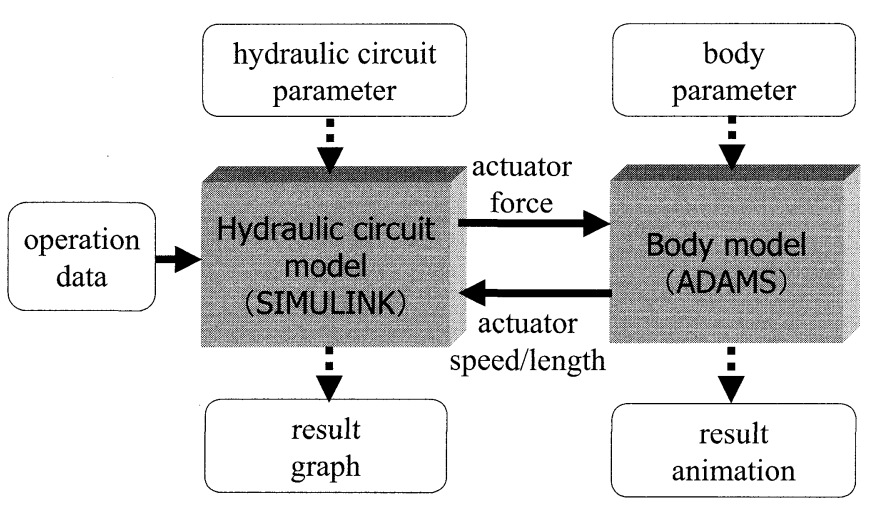

Figure 1 Structure of the simulation model 


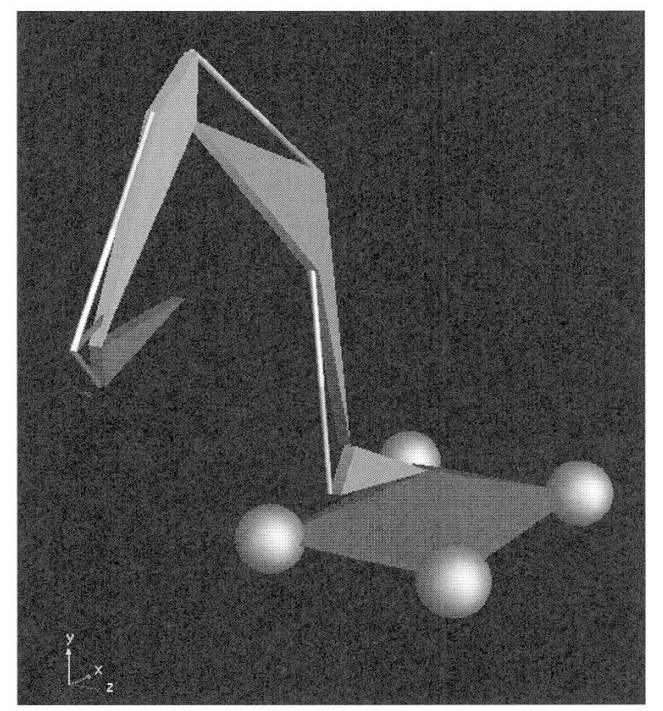

Figure 2 Body model

\section{DIGGING-TURNING-LOADING CYCLE ANALYSIS}

Figure 3 shows the block diagram of the simulation model.

In this simulation, the simulation model takes various characteristics into account, such as volumetric and mechanical efficiency of pump and motor, friction of cylinder, pressure loss of control valve inside passage and engine fuel consumption characteristic.

Figure 4(a)-4(f) shows simulation results.

Figure 4(a) shows results for the boom, namely (a1) pilot pressure for boom operation, (a2) pressure of boom cylinder bottom side, (a3) pressure of boom cylinder rod side, and (a4) length of boom cylinder.

Figure 4(b) shows results for the arm, namely (b1) pilot pressure for arm operation, (b2) pressure of arm cylinder bottom side, (b3) pressure of arm cylinder rod side, and (b4) length of arm cylinder.

Figure 4(c) shows results for the bucket, namely (c1) pilot pressure for bucket operation, (c2) pressure of bucket cylinder bottom side, (c3) pressure of bucket cylinder rod side, and (c4) length of bucket cylinder.

Figure 4(d) shows results for the swing, namely (d1) pilot pressure for swing operation, (d2) pressure of swing motor right side, (d3) pressure of swing motor left side, (d4) rotational speed of swing motor.

Figure 4(e) shows results for the pump, namely (e1) discharge pressure of pump-1, and (e2) discharge pressure of pump-2.

Figure 4(f) shows results for the engine, namely (f1) rotational speed of engine, and (f2) flow rate of fuel.

In these figures, the blue lines show experiment results whereas the red lines are simulation results.

The simulation result matches the experimental one with reasonable accuracy.

However, there are a few differences that are remarkable enough to notice at some parts of the results.

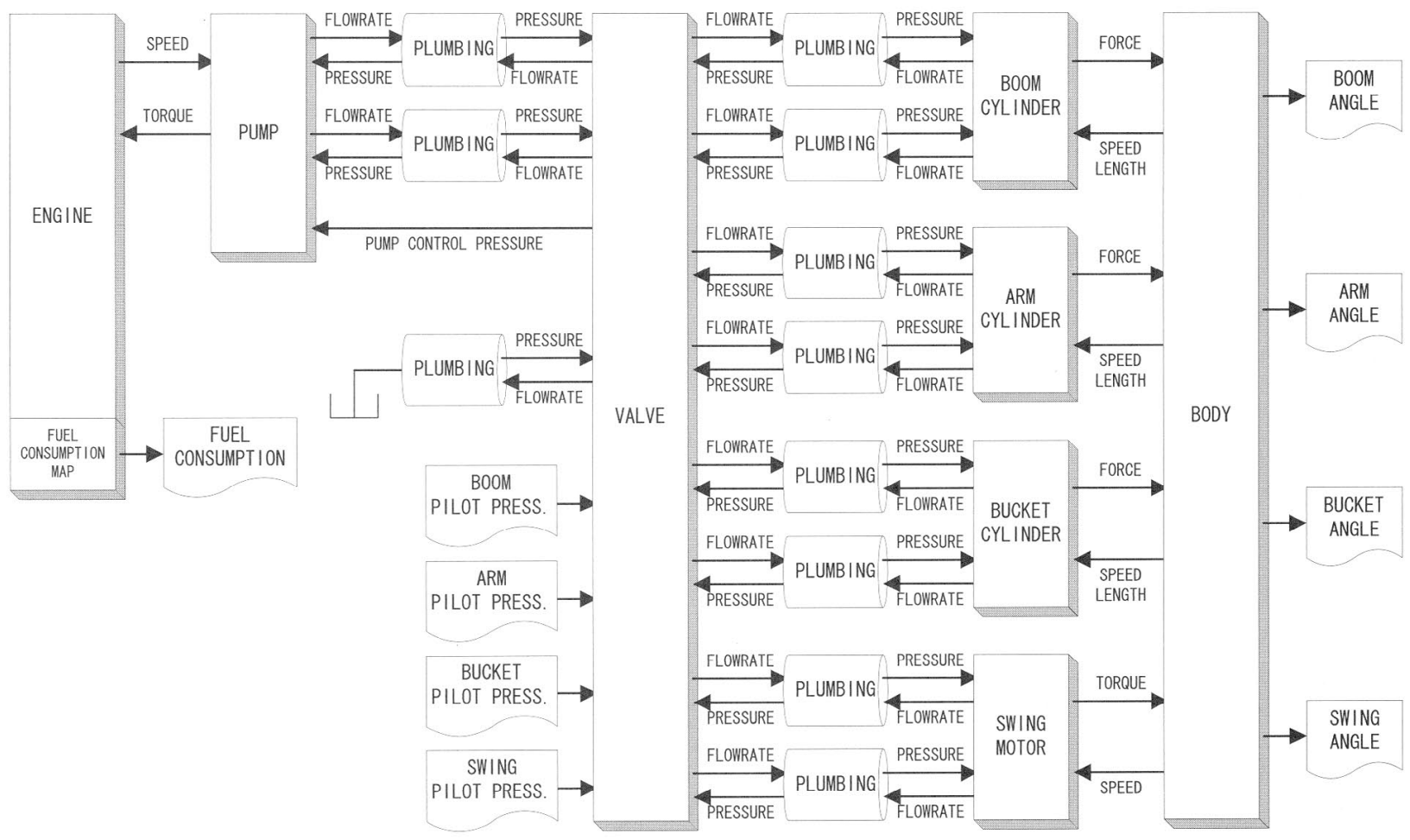

Figure 3 Block diagram of the simulation model 

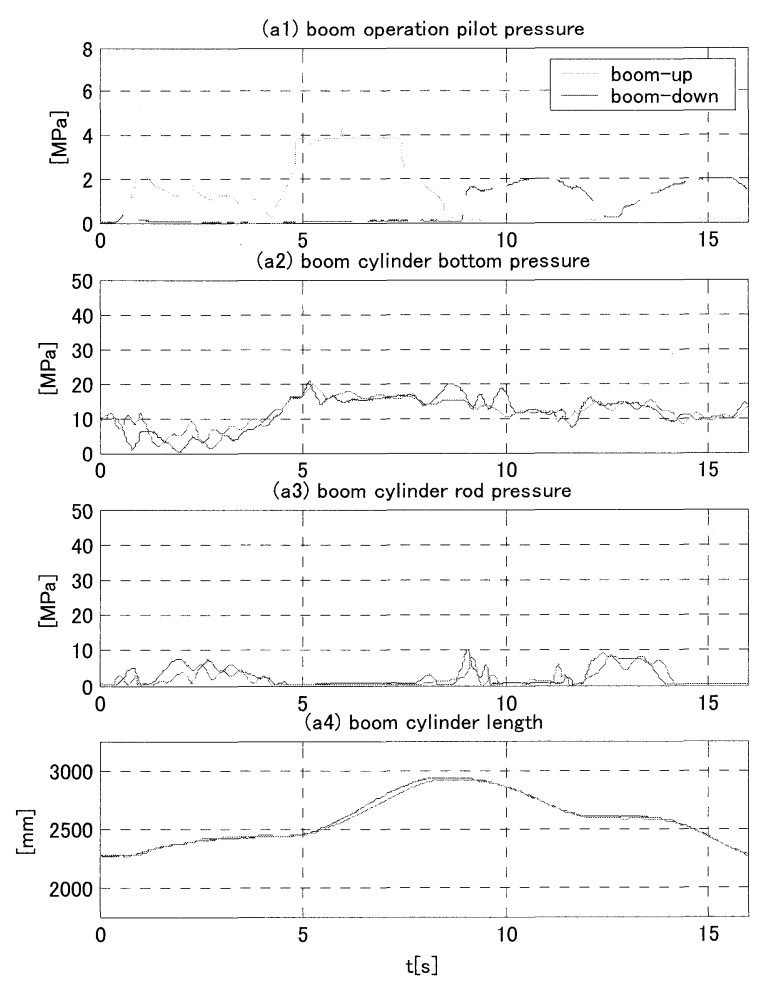

Figure 4(a) Simulation result (boom)
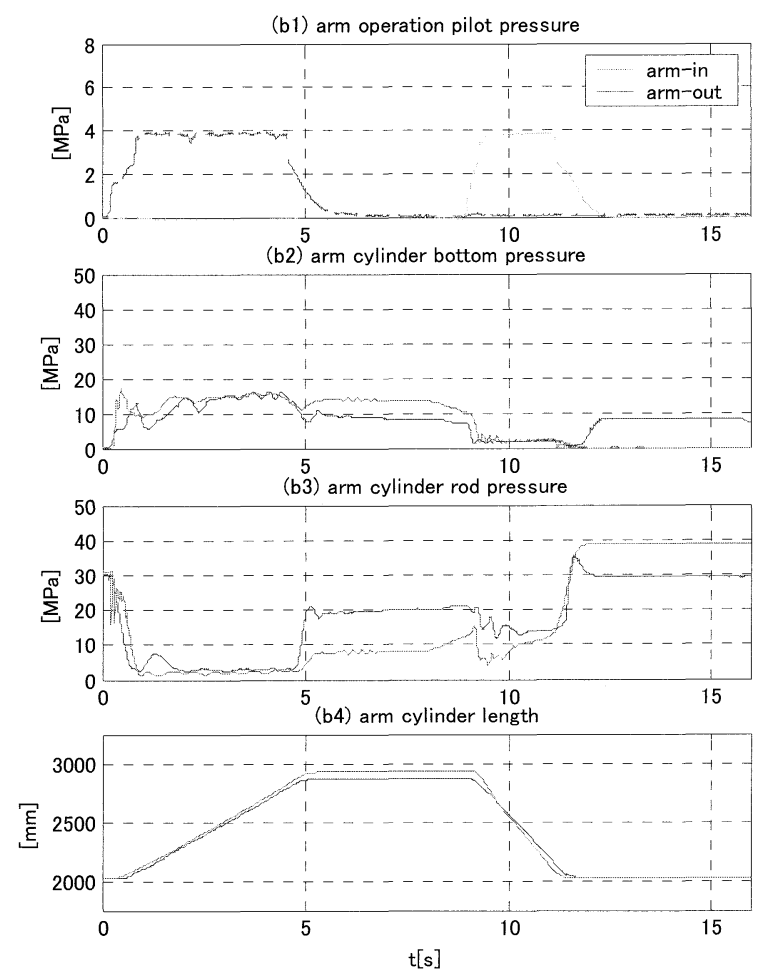

Figure 4(b) Simulation result (arm)
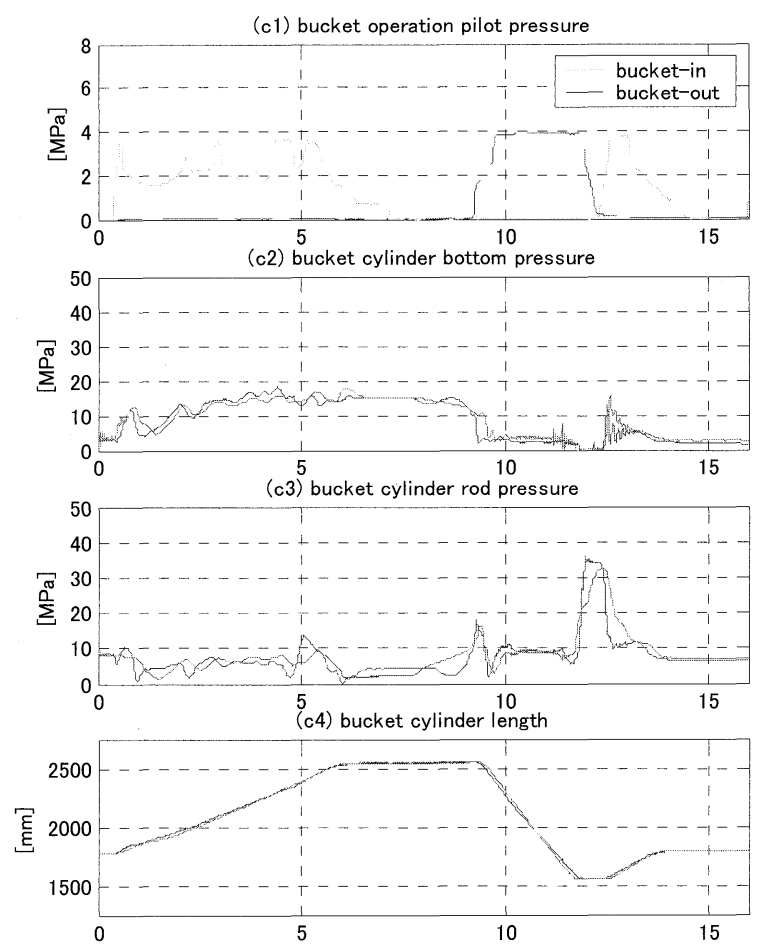

$\mathrm{t}[\mathrm{s}]$

Figure 4(c) Simulation result (bucket)
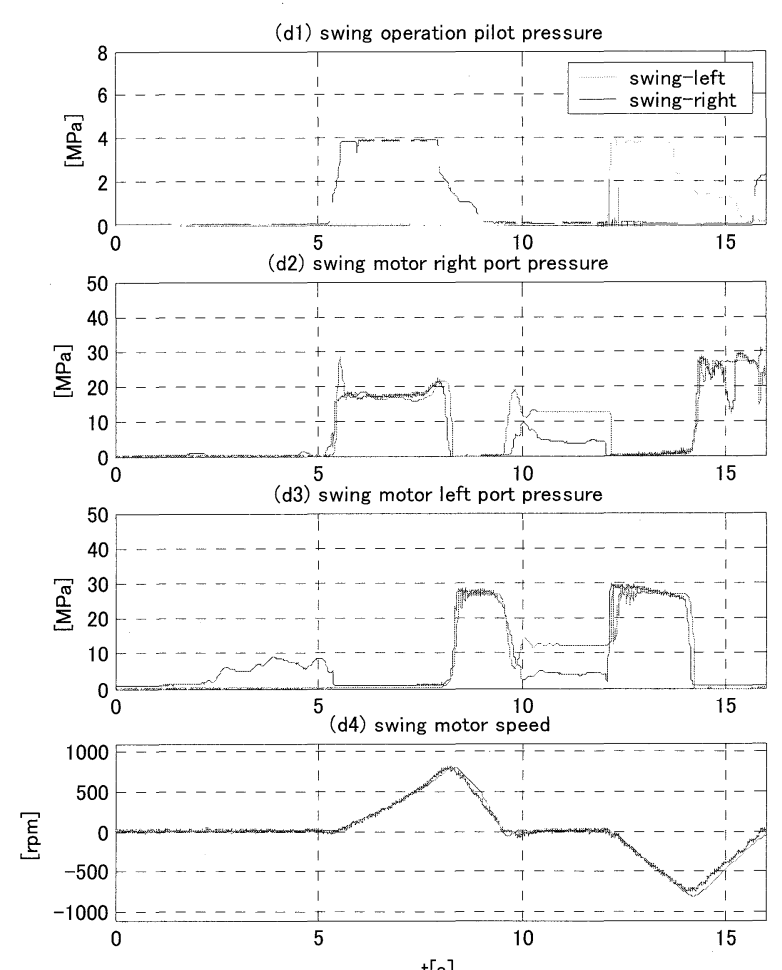

$\mathrm{t}[\mathrm{s}]$

Figure 4(d) Simulation result (swing) 

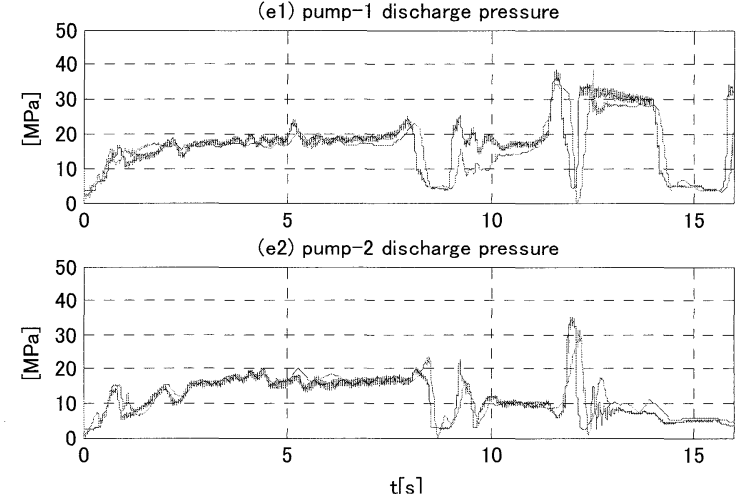

Figure 4(e) Simulation result (pump)
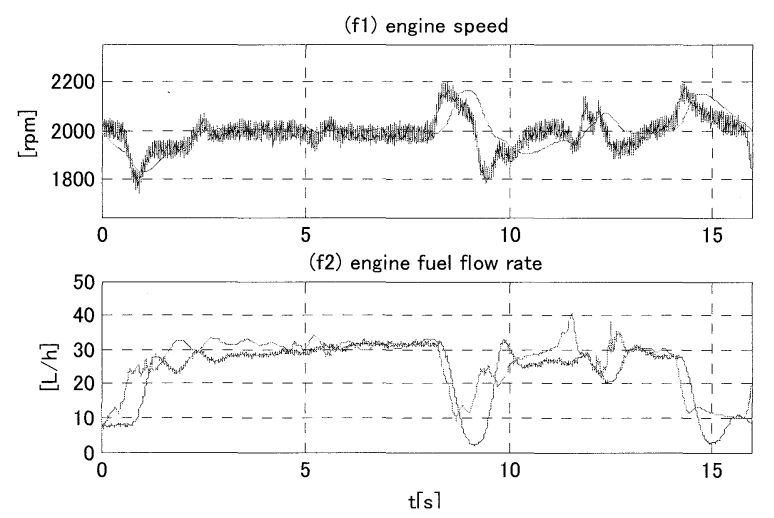

Figure 4(f) Simulation result (engine)

At first, differences are seen for time $=5-9$ seconds in (b2) pressure of arm cylinder bottom side and (b3) pressure of arm cylinder rod side, are known to be cause by error of (b4) length of arm cylinder. The error then affects body posture, and as a consequence, changes pressure to sustain gravity moments of the body.

Secondly, for the error for time $=12-16$ seconds, it is caused by relief pressure in the cylinder stroke end.

Thirdly, an error in (d3) pressure of swing motor left side, in time $=$ approximately $2.5-5$ seconds, is due to digging reaction force acts on the side face of bucket, which in fact is not considered nor modeled in this simulation.

Finally, for the errors found in (d2) pressure of swing motor right side and (d3) pressure of swing motor left side for time $=10-12.5$ seconds, they are known to be cause by a leak in the spool clearance when the valve is in its neutral position.

Using the simulation result, we calculate energy consumption during digging-turning-loading operation.

Figure 5 shows the result of the analysis, where total output energy is $100 \%$.

In the figure, a term "external output" means sum of output required to dig and output required to drive the body.

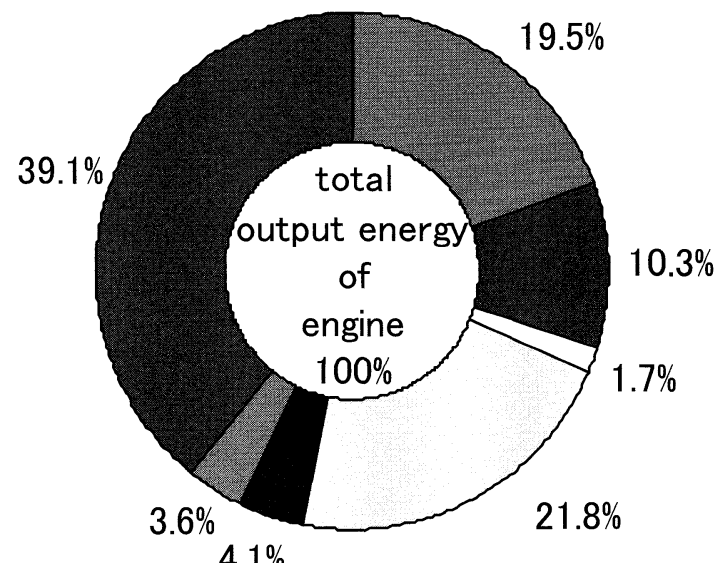

$4.1 \%$

loss of engine accessory(including pilot pump)

Dump loss

$\square$ loss of plumbing between pump and valve

$\square$ valve loss

loss of plumbing between valve and actuator

$\square$ actuator loss

国external output

Figure 5 Result of energy analysis

From the figure, it is cleared that valve internal loss is the largest contributor to the total energy consumed by the hydraulic components, and pump internal loss is the second.

By reducing internal loss of valve and pump, the total energy consumption could be saved.

\section{CONCLUSION}

Example of the system simulation for hydraulic excavator considering hydraulic circuit and a body movement was introduced.

We will use this simulation model for the product development effectively.

\section{REFERENCE}

1. Maehata K., System Simulation for Hydraulic Excavator, KYB TECHNICAL REVIEW, 2006, No.32, pp.4-9. 